\title{
ON THE ASYMPTOTIC BEHAVIOUR OF NONNEGATIVE SOLUTIONS OF A CERTAIN INTEGRAL INEQUALITY
}

BY

\author{
GUNNAR A. BROSAMLER
}

\begin{abstract}
The asymptotic behaviour of nonnegative solutions of a certain integral inequality is discussed, in the framework of a probabilistic-potential theoretic boundary theory.
\end{abstract}

1. Introduction. Let $\mu$ be a measure on $R$, the real line, such that

$$
\mu(\xi, \xi+1] \leqq 1, \quad \xi \in R .
$$

In the theory of slowing down of neutrons one is interested in bounded, or more generally, nonnegative solutions $f$ of the integral equation

$$
\int_{(\xi, \xi+1]} f(\eta) \mu(d \eta)=f(\xi), \quad \xi \in R
$$

(see [4]).

This equation and in particular the limiting behaviour of $f$ at $+\infty$ and $-\infty$ was studied by Slater and Wilf under the very restrictive assumption that $\mu(d \xi)=K(\xi) d \xi$, $K \uparrow 1$ [5]. In a subsequent paper [6], Slater replaced " $K \uparrow 1$ " by " $0<K \leqq 1$ ".

As was pointed out in [1], the natural approach to equation (1.2) is a potential theoretic-probabilistic one. This approach makes artificial assumptions on $\mu$ unnecessary, and allowed us to study-more generally-the nonnegative solutions of

$$
\int_{(\xi, \xi+1]} f(\eta) \mu(d \eta) \leqq f(\xi), \quad \xi \in R .
$$

Whereas one admits as solutions of (1.2) only real-valued functions, one allows for (1.3) extended-real-valued functions which are locally $\mu$-integrable. In [1] we obtained theorems on the structure of these solutions as well as theorems on their asymptotic behaviour at $+\infty$.

It is the purpose of this paper to prove theorems on the asymptotic behaviour of nonnegative solutions of (1.2) and (1.3) at $-\infty$ with no assumption on $\mu$ other than (1.1). In particular we shall prove the so-called "renewal" theorem on the

Received by the editors March 8, 1971.

AMS 1969 subject classifications. Primary 6062, 2652, 4510.

Key words and phrases. Linear integral equation, probabilistic boundary theory, approximate Markov chains, renewal theorem. 
asymptotic behaviour of the Green function $g$ of equation (1.2) which was introduced in [1]. The main results of this paper are listed in $\S 3 ; \S 2$ contains a review of the main results of [1].

In this paper an important role is played by the duals of (1.2), (1.3), namely

$$
\begin{aligned}
& \int_{[\xi-1, \xi)} f(\eta) \mu(d \eta)=f(\xi), \quad \xi \in R, \\
& \int_{[\xi-1, \xi)} f(\eta) \mu(d \eta) \leqq f(\xi), \quad \xi \in R .
\end{aligned}
$$

(Note that $\mu[\xi-1, \xi) \leqq 1$.)

A trivial remark first: Symmetry furnishes an obvious 1-1 correspondence between statements relating to (1.2), (1.3) and those relating to $(1.2)^{\wedge},(1.3)^{\wedge}$. We shall make frequent use of this trivial duality principle.

Less superficial is a connection between (1.2), (1.3) and $(1.2)^{\wedge},(1.3)^{\wedge}$ which is suggested by the potential theoretic point of view: If we define the substochastic transition function $P$ on $R$ by

$$
P(\xi, A)=\mu\{(\xi, \xi+1] \cap A\},
$$

$\xi \in R, A$ Borel $\subseteq R$, then the nonnegative $P$-regular and $P$-superregular functions are just the nonnegative solutions of (1.2) and (1.3), whereas the $\mu$-densities of the $P$-regular and $P$-superregular measures (see [1]) which are absolutely continuous with respect to $\mu$ (note: a $P$-regular measure is automatically absolutely continuous with respect to $\mu$ ) are essentially the nonnegative solutions of $(1.2)^{\wedge}$ and $(1.3)^{\wedge}$. If instead we consider the potential theory of the substochastic transition function

$$
\hat{P}(\xi, A)=\mu\{[\xi-1, \xi) \cap A\},
$$

$\xi \in R, A$ Borel $\subseteq R$, the roles of (1.2), (1.3) and (1.2)^, (1.3)^ are obviously interchanged. In any case, it is not surprising that at a certain stage a simultaneous study of (super-)regular functions and (super-)regular measures should become inevitable.

The results obtained in [1] were put into the framework of a boundary theory for the transition function $P$. In particular, we identified-under condition (A) (see \$2) -the Martin exit boundary as $\{+\infty\}$ and obtained versions of the Riesz-Martin representation and Fatou limit theorems for nonnegative $P$-superregular functions. In the light of the above, the theorems of this paper complete the boundary theory of the $P$-potential theory: The Martin entrance boundary can be identified-under condition $(\hat{\mathrm{A}})($ see $\S 2)$-as $\{-\infty\}$ and our limit theorems provide limit theorems for the nonnegative $P$-superregular functions at this boundary. In short, [1] and the present paper present one of the very few examples of probabilistic potential theory, whose boundary theory is worked out completely. 
We conclude with a few technical remarks: The proofs given in [1] were strictly potential theoretic; their probabilistic interpretation could be given in terms of Markov chains with transition function $P$ and varying starting points $\xi \in R$. For the proof of the main theorem (i.e. Theorem (3.1)) in this paper, however, we need a probabilistic argument, involving the concept of an approximate Markov chain in the sense of Hunt [2] and time reversal. With this in mind, we emphasize the probabilistic nature of the proof of our renewal theorem.

2. Results from [1] and their duals. We start by recalling some notations and results from [1]. For $\xi \in R, A$ Borel $\subseteq R$, let $P_{0}(\xi, A)=\chi_{A}(\xi)$,

$$
P_{n+1}(\xi, A)=\int P_{n}(\eta, A) P(\xi, d \eta)
$$

for $n \geqq 0, G(\xi, A)=\sum_{n=0}^{\infty} P_{n}(\xi, A)$ (Green function). For $f \geqq 0$, let $\left(P_{n} f\right)(\xi)=$ $\int f(\eta) P_{n}(\xi, d \eta)$ and let $\psi=\downarrow \lim _{n \rightarrow \infty} P_{n} 1$. We have

$$
G(\xi, A)=\chi_{A}(\xi)+\int_{A} g(\xi, \eta) \mu(d \eta)
$$

with $g(\xi, \eta)=G(\xi,[\eta-1, \eta))$. Moreover, $0 \leqq g \leqq 4, g(\xi, \eta)=0$ for $\xi \geqq \eta$, and $\psi(\xi)$ $\leqq g(\xi, \eta)$ for $\xi<\eta-1$.

As we mentioned in $\S 1$, the nonnegative solutions of (1.2) and (1.3) are just the nonnegative $P$-regular and $P$-superregular functions. One has the following results on these functions:

(1) Every $P$-superregular function $u \geqq 0$ has a unique decomposition into the sum of a nonnegative $P$-regular function and a $P$-potential

$$
v(\xi)=\int(u-P u)(\eta) G(\xi, d \eta)
$$

(Riesz decomposition).

(2) $\psi \neq 0$ iff

(A)

$$
\text { (i) } \limsup _{\xi \rightarrow \infty} \mu(\xi, \xi+1]>0 \text {, }
$$

$$
\text { (ii) } \int^{\infty}\{1-\mu(\xi, \xi+1]\} \mu(d \xi)<\infty \text {. }
$$

(3) If (A) holds, all nonnegative $P$-regular functions are proportional to $\psi$.

(4) If (A) holds, $\lim _{\xi \rightarrow \infty} \psi(\xi)=1$.

(5) If (A) does not hold, a nonnegative $P$-regular function $\not \equiv 0$ has limit $\infty$ at $+\infty$.

(6) If $v$ is a $P$-potential satisfying the condition $\int^{\infty}(v-P v)(\eta) \mu(d \eta)<\infty$, then $\lim _{\xi \rightarrow \infty} P v(\xi)=0$. If (A) holds, this condition is always satisfied, and moreover, $\lim _{\xi \rightarrow \infty ; \xi \notin A} v(\xi)=0$ for some Borel set $A \subseteq R$ such that $\int^{\infty} \chi_{A}(\xi) \mu(d \xi)<\infty$.

For details we refer to [1]. 
We can obviously dualize the statements (1)-(6) by replacing $P, G, \psi, \lim _{\xi \rightarrow+\infty}$, $\int^{\infty}$ by $\hat{P}, \hat{G}=\sum_{n=0}^{\infty} \hat{P}_{n}, \hat{\psi}=\downarrow \lim \hat{P}_{n} 1, \lim _{\xi \rightarrow-\infty}, \int_{-\infty}$ and condition (A) by condition

$$
\text { (i) } \limsup _{\xi \rightarrow-\infty} \mu[\xi-1, \xi)>0 \text {, }
$$

$$
\text { (ii) } \int_{-\infty}\{1-\mu[\xi-1, \xi)\} \mu(d \xi)<\infty \text {. }
$$

The trivial duality principle involved was already mentioned in $\$ 1$. The subsequently mentioned connection between $P$-(super-)regular measures and $\hat{P}$-(super-) regular functions is reflected by the following fact: We have, for $\hat{g}(\xi, \eta)=$ $\hat{G}(\xi,(\eta, \eta+1])$,

$$
\hat{g}(\xi, \eta)=g(\eta, \xi)
$$

as is easily verified.

We conclude this section with a probabilistic interpretation of $\psi[\hat{\psi}]$. If $\left(\Omega, \mathfrak{A}, \operatorname{Pr}_{\xi} ; X_{n}, n \geqq 0\right)$ is a Markov chain with substochastic transition function $P[P]$ and starting point $\xi \in R$, then this chain has no cluster points in $R$ and $\psi(\xi)$ $=\operatorname{Pr}_{\xi}\left\{\lim _{n \rightarrow \infty} X_{n}=\infty\right\}\left[\hat{\psi}(\xi)=\operatorname{Pr}_{\xi}\left\{\lim _{n \rightarrow \infty} X_{n}=-\infty\right\}\right]$.

3. Main theorems. As we shall see in the next section, condition (A) implies the existence of $\alpha=\lim _{\xi \rightarrow \infty} \int_{(\xi, \xi+1]} \mu\{\eta\} \mu(d \eta)$ and dually, condition $(\hat{\mathrm{A}})$ the existence of $\hat{\alpha}=\lim _{\xi \rightarrow-\infty} \int_{[\xi-1, \xi)} \mu\{\eta\} \mu(d \eta)$. We state now the main results of this paper.

(3.1) THEOREM. Let h be a nonnegative, finite, measurable function on $(-\infty, M+1]$ such that $P h=h$ on $(-\infty, M]$. Then $\lim _{\xi \rightarrow-\infty} h(\xi)$ exists. This limit is 0 if $(\hat{\mathrm{A}})$ does not hold.

(3.2) Corollary. (1) $\psi(-\infty)=\lim _{\xi \rightarrow-\infty} \psi(\xi)$ exists.

(2) $\psi(-\infty)=0$ if $(\hat{\mathrm{A}})$ does not hold.

(3.3) Proposition. If (A) holds or equivalently if $\psi \equiv 0$, then

$$
\begin{aligned}
\int_{-\infty}^{+\infty} \psi(\xi)\{1-\mu[\xi-1, \xi)\} \mu(d \xi) & =\frac{1+\alpha}{2} \psi(+\infty)-\frac{1+\hat{\alpha}}{2} \psi(-\infty) \text { or } \\
& =\frac{1+\alpha}{2} \psi(+\infty)
\end{aligned}
$$

according to whether $(\hat{\mathrm{A}})$ holds or not.

(3.4) TheORem (Renewal TheORem). (1) $\lim _{\eta \rightarrow+\infty} g(\xi, \eta)=(2 /(1+\alpha)) \psi(\xi)$ or 0 according to whether (A) holds or not.

(2) $\lim _{\xi \rightarrow-\infty} g(\xi, \eta)=(2 /(1+\hat{\alpha})) \hat{\psi}(\eta)$ or 0 according to whether $(\hat{\mathrm{A}})$ holds or not. 
(3.5) TheOrem. Let $v=G f$ be a P-potential, where $f=v-P v \geqq 0$.

(1) If $(\hat{\mathrm{A}})$ does not hold, and $\int f(\eta) \mu(d \eta)<\infty$, then $\lim _{\xi \rightarrow-\infty} P v(\xi)=0$.

(2) If $(\hat{\mathrm{A}})$ holds, and $\int_{-\infty} f(\eta) \mu(d \eta)=\infty$, then $\lim _{\xi \rightarrow-\infty} v(\xi)=\lim _{\xi \rightarrow-\infty} P v(\xi)=\infty$.

(3) If (Â) holds, and $\int_{-\infty} f(\eta) \mu(d \eta)<\infty$, then

$$
\lim _{\xi \rightarrow-\infty ; \xi \notin A} v(\xi)=\lim _{\xi \rightarrow-\infty} P v(\xi)=\frac{2}{1+\hat{\alpha}} \int \hat{\psi}(\eta) f(\eta) \mu(d \eta)<\infty,
$$

where $A$ is some Borel set $\subseteq R$ such that $\int_{-\infty} \chi_{A}(\xi) \mu(d \xi)<\infty$.

Again, one can obviously dualize the preceding results by making the substitutions mentioned in $\S 2$ and by replacing in addition $(\hat{\mathrm{A}}), \alpha, \hat{\alpha}, \lim _{\xi \rightarrow-\infty},(-\infty, M]$, $\mu[\xi-1, \xi)$, by (A), $\hat{\alpha}, \alpha, \lim _{\xi \rightarrow+\infty},[-M, \infty), \mu(\xi, \xi+1]$. In view of (2.3) the two parts of Theorem (3.4) are duals of each other, and it is hence sufficient to prove only part (2). The following theorem relates $\psi(-\infty)$ and $\hat{\psi}(+\infty)$.

(3.6) THEOREM. $\psi(-\infty)=0$ iff $\hat{\psi}(+\infty)=0$. If the limits are not 0 , then $\hat{\psi}(+\infty) / \psi(-\infty)=(1+\hat{\alpha}) /(1+\alpha)$.

The proofs are given in $\S 6$. Probabilistic aspects are also mentioned in $\S 5$ (Remarks).

4. Preliminaries. In this section we shall discuss the conditions (A) and ( $\hat{A})$. Firstly we remark that if condition (A)(ii) holds, then (A)(i) is equivalent to

$$
\lim _{\xi \rightarrow \infty} \mu(\xi, \xi+1]=1
$$

This follows from $\psi(\xi) \leqq \mu(\xi, \xi+1] \leqq 1$, and $\lim _{\xi \rightarrow \infty} \psi(\xi)=1$ if (A) holds. Dually, under condition $(\hat{\mathrm{A}})(\mathrm{ii}),(\hat{\mathrm{A}})(\mathrm{i})$ is equivalent to

$$
\lim _{\xi \rightarrow-\infty} \mu[\xi-1, \xi)=1
$$

We proceed to the following

(4.1) Lemma. Let $f \geqq 0$ be a locally $\mu$-integrable function on $R$. Then for $M, N \in R$, $M+1<N$,

$$
\begin{aligned}
\int_{(M, N)}\{1-\hat{P} 1\}(\xi) f(\xi) \mu(d \xi)= & \int_{(M, N-1)}\{f-P f\}(\xi) \mu(d \xi) \\
& -\int_{(M-1, M]} \mu(d \xi) \int_{(M, \xi+1]} f(\eta) \mu(d \eta)+(\hat{P} f)(N) \\
& -\int_{[N-1, N)} \mu(d \xi) \int_{(\xi, N)} f(\eta) \mu(d \eta) .
\end{aligned}
$$


Proof. By application of Fubini's theorem to

$$
\int_{(M, N)}(\hat{P} 1)(\xi) f(\xi) \mu(d \xi)=\int\left(\int \chi_{[\xi-1, \xi)}(\eta) \chi_{(M, N)}(\xi) f(\xi) \mu(d \eta)\right) \mu(d \xi)
$$

(4.2) Proposition. Condition (A)(ii) is equivalent to

(A) (ii')

$$
\int^{\infty}\{1-\mu[\xi-1, \xi)\} \mu(d \xi)<\infty
$$

Proof. If we apply Proposition (4.1) to $f \equiv 1$, we have

$$
\begin{aligned}
\int_{(M, N)}\{1-\mu[\xi-1, \xi)\} \mu(d \xi)= & \int_{(M, N-1)}\{1-\mu(\xi, \xi+1]\} \mu(d \xi) \\
& -\int_{(M-1, M]} \mu(M, \xi+1] \mu(d \xi)+\mu[N-1, N) \\
& -\int_{(N-1, N)} \mu(\xi, N) \mu(d \xi) .
\end{aligned}
$$

The assertion of the proposition follows.

(4.4) Proposition. Assume that condition (A) holds. Then

$$
\alpha=\lim _{\xi \rightarrow \infty} \int_{(\xi, \xi+1]} \mu\{\eta\} \mu(d \eta)
$$

exists. Moreover,

$$
\begin{aligned}
& \lim _{\xi \rightarrow \infty} \int_{[\xi, \xi+1)} \mu(\eta, \xi+1) \mu(d \eta)=\frac{1}{2}(1-\alpha), \\
& \lim _{\xi \rightarrow \infty} \int_{[\xi, \xi+1)} \mu[\eta-1, \xi) \mu(d \eta)=\frac{1}{2}(1+\alpha) .
\end{aligned}
$$

Proof. If (A) holds, we have $\lim _{\xi \rightarrow \infty} \mu(\xi, \xi+1]=1$, hence $\lim _{\xi \rightarrow \infty} \mu[\xi, \xi+1)$ $=1$, and we may conclude from (4.3) and Proposition (4.2) that

$$
\lim _{\xi \rightarrow \infty} \int_{[\xi, \xi+1)} \mu(\eta, \xi+1) \mu(d \eta)
$$

exists. Now we have by Fubini's theorem.

$$
\int_{[\xi, \xi+1)} \mu(\eta, \xi+1) \mu(d \eta)=\int_{[\xi, \xi+1)} \mu[\xi, \eta) \mu(d \eta),
$$

and, therefore,

$$
\begin{aligned}
\{\mu[\xi, \xi+1)\}^{2} & =\int_{[\xi, \xi+1)} \mu[\xi, \xi+1) \mu(d \eta) \\
& =2 \int_{[\xi, \xi+1)} \mu(\eta, \xi+1) \mu(d \eta)+\int_{[\xi, \xi+1)} \mu\{\eta\} \mu(d \eta)
\end{aligned}
$$


This equation implies the existence of

$$
\lim _{\xi \rightarrow \infty} \int_{[\xi, \xi+1)} \mu\{\eta\} \mu(d \eta)=\lim _{\xi \rightarrow \infty} \int_{(\xi, \xi+1]} \mu\{\eta\} \mu(d \eta)
$$

-which we denote by $\alpha$ - as well as the identification

$$
\lim _{\xi \rightarrow \infty} \int_{[\xi, \xi+1)} \mu(\eta, \xi+1) \mu(d \eta)=\frac{1}{2}(1-\alpha)
$$

We now conclude the last statement of the proposition from the preceding two, since

$$
\lim _{\xi \rightarrow \infty}\left\{\int_{[\xi, \xi+1)} \mu[\eta-1, \xi) \mu(d \eta)-\int_{[\xi, \xi+1)} \mu[\eta, \xi+1) \mu(d \eta)\right\}=0,
$$

as can be seen from $\mu[\eta-1, \xi)-\mu[\eta, \xi+1)=\mu[\eta-1, \eta)-\mu[\xi, \xi+1)$ for $\eta \in[\xi, \xi+1)$, and $\lim _{\xi \rightarrow \infty} \mu[\xi, \xi+1)=1$.

REMARK. Obviously Propositions (4.2) and (4.4) have their duals. Thus (Â)(ii) is equivalent to

$$
\int_{-\infty}\{1-\mu(\xi, \xi+1]\} \mu(d \xi)<\infty
$$

Now assume that $(\hat{\mathrm{A}})$ holds. Then

$$
\hat{\alpha}=\lim _{\xi \rightarrow-\infty} \int_{(\xi-1, \xi]} \mu\{\eta\} \mu(d \eta)=\lim _{\xi \rightarrow-\infty} \int_{[\xi-1, \xi)} \mu\{\eta\} \mu(d \eta)
$$

exists, and

$$
\begin{aligned}
& \lim _{\xi \rightarrow-\infty} \int_{(\xi-1, \xi]} \mu(\xi-1, \eta) \mu(d \eta)=\frac{1}{2}(1-\hat{\alpha}), \\
& \lim _{\xi \rightarrow-\infty} \int_{(\xi-1, \xi]} \mu(\xi, \eta+1] \mu(d \eta)=\frac{1}{2}(1+\hat{\alpha}) .
\end{aligned}
$$

5. An approximate Markov chain. The probabilistic tool used in this paper is an "approximate Markov chain with transition function $P$ and sojourn measure $\mu$ ”. In $\S 6$ we shall use this tool via Theorems (5.1) and (5.2) to prove Theorem (3.1).

The concept of approximate Markov chain was introduced by Hunt [2] for transient substochastic transition functions and associated superregular measures, on a countable state space. In our case, we have as transition function the function $P$, defined on the real line, rather than on a countable set, and as $P$-superregular measure the measure $\mu$. Our Theorem (5.1) is (essentially) a transcription of Theorem (10.9) in [3], and our Theorem (5.3) one of Theorem (1.1) in [2]. Proofs of these two theorems will be omitted since they too are obtained by simple transcriptions of the corresponding proofs in [3], [2]. (Two minor errors in the proof of Theorem (10.9) in [3] are easily corrected.) May it suffice to mention, that the transcriptions are made possible by fixin' $\mu$ as a reference measure on $R$; this allows one-when dealing with measures on $R$ that have $\mu$-densities-to deal with 
their densities instead; in particular we have for the $P$-superregular measure $\mu$ the $\hat{P}$-superregular density 1 , and for the measures $P(\xi, \cdot)$ and $\hat{P}(\xi, \cdot)$ the densities $p(\xi, \eta)=\chi_{(\xi, \xi+1]}(\eta)$ and $\hat{p}(\xi, \eta)=\chi_{[\xi-1, \xi)}(\eta)=1 \cdot p(\eta, \xi) / 1$.

Unlike Theorems (5.1) and (5.3), Theorems (5.2), (5.4), (5.5) are dependent on the special nature of our transition function.

We shall start now with the existence theorem. For this purpose let $S=R \cup\{\sigma, \rho\}$ ( $\sigma, \rho$ two different fictitious states), endowed with the obvious $\sigma$-field structure, and let $P^{\prime}$ be the stochastic extension of $P$ to $R \cup\{\rho\}$ (see [1]).

(5.1) THeOREM. There exists a (not necessarily finite) measure space ( $\Omega, \mathfrak{A}, \operatorname{Pr})$ and measurable mappings $Z_{k}: \Omega \rightarrow S,-\infty<k<+\infty$, such that

(1) On an $\omega$-set of full Pr-measure,

$Z_{k}(\omega)=\sigma$ implies $Z_{m}(\omega)=\sigma$ for $m \leqq k$.

$Z_{k}(\omega)=\rho$ implies $Z_{m}(\omega)=\rho$ for $m \geqq k$.

There exists $k(\omega)$ such that $Z_{k(\omega)}(\omega) \in R$.

(2) If for any bounded Borel set $B \subseteq R$, we let $\Omega_{B}=\bigcup_{k=-\infty}^{+\infty} Z_{k}^{-1}(B)$ and $\tau_{B}(\omega)$ $=\inf \left\{k ; Z_{k}(\omega) \in B\right\}$ for $\omega \in \Omega_{B}$, then $\tau_{B}>-\infty$, Pr-a.e. on $\Omega_{B}$ and the process $X_{k}^{(B)}=Z_{k+\tau_{B}}, k \geqq 0$, defined on $\Omega_{B}$, is a “Markov chain" with transition function $P^{\prime}$ and finite initial distribution.

(3) For any Borel set $A \subseteq R$,

$$
\int_{k=-\infty}^{+\infty} \chi_{A}\left(Z_{k}(\omega)\right) d \operatorname{Pr}(\omega)=\mu(A)
$$

Any process $(\Omega, \mathfrak{A}, \operatorname{Pr}),\left\{Z_{k}: \Omega \rightarrow S,-\infty<k<+\infty\right\}$ having the properties of the preceding theorem is called an approximate Markov chain with transition function $P$ and sojourn measure $\mu$. Next we discuss some properties of such a chain. For this purpose let $\beta=\frac{1}{2}(1+\alpha)$ or 0 according to whether (A) holds or does not hold; dually let $\hat{\beta}=\frac{1}{2}(1+\hat{\alpha})$ or 0 according to whether $(\hat{\mathrm{A}})$ holds or does not hold.

(5.2) ThEOREM. Let $(\Omega, \mathfrak{A}, \operatorname{Pr}),\left\{Z_{k}: \Omega \rightarrow S,-\infty<k<+\infty\right\}$ be an approximate Markov chain with transition function $P$ and sojourn measure $\mu$. Then

(1) On an w-set of full Pr-measure,

(a) For all $k, Z_{k}(\omega), Z_{k+1}(\omega) \in R$ implies $Z_{k}(\omega)<Z_{k+1}(\omega) \leqq Z_{k}(\omega)+1$.

(b) The set $\left\{Z_{k}(\omega),-\infty<k<+\infty\right\}$ has no cluster points in $R$.

(2) For $M \in R$,

$$
\operatorname{Pr}\left\{\Omega_{(-\infty, M]}\right\}=\int_{(-\infty, M]}\{1-\mu[\xi-1, \xi)\} \mu(d \xi)+\hat{\beta}
$$

and

$$
\operatorname{Pr}\left\{\Omega_{[M,+\infty)}\right\}=\int_{[M, \infty)}\{1-\mu(\xi, \xi+1]\} \mu(d \xi)+\beta .
$$


(3) (a) $\operatorname{Pr}\left\{\lim _{k \rightarrow-\infty} Z_{k}=-\infty\right\}=\hat{\beta}, \operatorname{Pr}\left\{\lim _{k \rightarrow+\infty} Z_{k}=+\infty\right\}=\beta$.

(b) $\operatorname{Pr}\left\{\lim _{k \rightarrow-\infty} Z_{k}=-\infty, \lim _{k \rightarrow+\infty} Z_{k}=+\infty\right\}=\hat{\beta} \psi(-\infty)=\beta \hat{\psi}(+\infty)$.

(4) 0-1-law. For any Borel set $A \subseteq R$,

$$
\begin{aligned}
\operatorname{Pr}\left\{Z_{k} \in A \text { i.o. as } k \rightarrow-\infty\right\} & =0 \text { if } \int_{-\infty} \chi_{A}(\xi) \mu(d \xi)<\infty, \\
& =\hat{\beta} \quad \text { if } \int_{-\infty} \chi_{A}(\xi) \mu(d \xi)=\infty ; \\
\operatorname{Pr}\left\{Z_{k} \in A \text { i.o. as } k \rightarrow+\infty\right\} & =0 \text { if } \int^{+\infty} \chi_{A}(\xi) \mu(d \xi)<\infty, \\
& =\beta \quad \text { if } \int^{+\infty} \chi_{A}(\xi) \mu(d \xi)=\infty
\end{aligned}
$$

(4') 0-1-law. If $\mathfrak{B}\left(\ldots, Z_{k-1}, Z_{k}\right)$ and $\mathfrak{B}\left(Z_{k}, Z_{k+1}, \ldots\right)$ are the sub- $\sigma$-fields of $\mathfrak{A}$, generated by $\left\{\ldots, Z_{k-1}, Z_{k}\right\}$ and $\left\{Z_{k}, Z_{k+1}, \ldots\right\}$ respectively, then for $\Lambda \in$ $\lim _{k \rightarrow-\infty} \mathfrak{B}\left(\ldots, Z_{k-1}, Z_{k}\right)$,

$$
\operatorname{Pr}\left\{\Lambda ; \lim _{k \rightarrow-\infty} Z_{k}=-\infty\right\}=0 \text { or } \hat{\beta}
$$

and for $\Lambda \in \lim _{k \rightarrow+\infty} \mathfrak{B}\left(Z_{k}, Z_{k+1}, \ldots\right)$,

$$
\operatorname{Pr}\left\{\Lambda ; \lim _{k \rightarrow+\infty} Z_{k}=+\infty\right\}=0 \text { or } \beta
$$

The proof of this theorem is very straightforward and we shall content ourselves to mention that it uses

(i) Results obtained in [1] for standard Markov chains with transition function $P^{\prime}$ and varying starting points $\xi \in R$.

(ii) Dual results for standard Markov chains with transition function $\hat{P}^{\prime}$ (=stochastic extension of $\hat{P}$ ) and varying starting points $\xi \in R$.

(iii) The fact that for any interval $B=(M, N) \subseteq R$, the process $X_{k}^{(B)}=Z_{k+\tau_{B}}$, $k \geqq 0$, defined on $\Omega_{B}$, is a "Markov chain" with transition function $P^{\prime}$ and initial distribution

$\operatorname{Pr}\left\{Z_{\imath_{B}} \in A ; \Omega_{B}\right\}=\mu^{B}(I-P)(A)$

$$
=\int_{(M+1, N)}\{1-\mu[\xi-1, \xi)\} \chi_{A}(\xi) \mu(d \xi)+\int_{(M, M+1]}\{1-\mu(M, \xi)\} \chi_{A}(\xi) \mu(d \xi) .
$$

( $\mu^{B}$ is the reduced measure of the $P$-superregular measure $\mu$ with respect to $B$.)

(iv) The fact that for any interval $B=(M, N) \subseteq R$, the process

$$
\begin{aligned}
X_{k}^{(B) \prime} & =Z_{-k+\zeta_{B}} & & \text { if } Z_{-k+\zeta_{B}} \in R, \\
& =\rho & & \text { if } Z_{-k+\zeta_{B}}=\sigma,
\end{aligned}
$$


defined on $\Omega_{B}$, with $\zeta_{B}(\omega)=_{\text {def }} \sup \left\{k ; Z_{k} \in B\right\}<\infty$ a.e. on $\Omega_{B}$, is a "Markov chain" with transition function $\hat{P}^{\prime}$ and initial distribution

$$
\begin{aligned}
\operatorname{Pr} & \left\{Z_{\zeta_{B}} \in A ; \Omega_{B}\right\} \\
& =\int_{(M, N-1)}\{1-\mu(\xi, \xi+1]\} \chi_{A}(\xi) \mu(d \xi)+\int_{[N-1, N)}\{1-\mu(\xi ; N)\} \chi_{A}(\xi) \mu(d \xi) .
\end{aligned}
$$

Obviously, item (iv) can be reformulated as

(5.3) THEOREM. Let $(\Omega, \mathfrak{A}, \operatorname{Pr}) ;\left\{Z_{k}: \Omega \rightarrow S,-\infty<k<+\infty\right\}$ be an approximate Markov chain with transition function $P$ and sojourn measure $\mu$. Then $(\Omega, \mathfrak{A}, \operatorname{Pr})$; $\left\{Z_{k}^{\prime}: \Omega \rightarrow S,-\infty<k<+\infty\right\}$ with

$$
\begin{aligned}
Z_{k}^{\prime}(\omega) & =Z_{-k}(\omega) & & \text { if } Z_{-k}(\omega) \in R, \\
& =\sigma & & \text { if } Z_{-k}(\omega)=\rho, \\
& =\rho & & \text { if } Z_{-k}(\omega)=\sigma,
\end{aligned}
$$

is an approximate Markov chain with transition function $\hat{P}$ and sojourn measure $\mu$, i.e. fulfills (1), (2), (3) of Theorem (5.1) with $P$ replaced by $\hat{P}$.

(This theorem makes precise the role of $\hat{P}$ as transition function of the "reversed process.")

We add some remarks to Theorem (5.2).

REMARK 1. By (5.2)(2), we have

$$
\begin{aligned}
& \operatorname{Pr}\left\{\Omega_{(-\infty, M]}\right\}<\infty \quad \text { iff }(\hat{\mathrm{A}}) \text { holds, } \\
& \operatorname{Pr}\left\{\Omega_{[M,+\infty)}\right\}<\infty \text { iff (A) holds, } \\
& \operatorname{Pr}\{\Omega\}<\infty \quad \text { iff }(\mathrm{A}) \text { and }(\hat{\mathrm{A}}) \text { hold. }
\end{aligned}
$$

REMARK 2. By (5.2)(3)(a), we have

$$
\begin{aligned}
& \operatorname{Pr}\left\{\lim _{k \rightarrow-\infty} Z_{k}=-\infty\right\}>0 \text { iff (Â) holds, } \\
& \operatorname{Pr}\left\{\lim _{k \rightarrow+\infty} Z_{k}=+\infty\right\}>0 \text { iff (A) holds. }
\end{aligned}
$$

REMARK 3. In view of Remark 2, (5.2)(1) and (5.2)(4') explain the use of the names "entrance" and "exit boundary" for $\{-\infty\}$ and $\{+\infty\}$ if $(\hat{\mathrm{A}})$ and $(\mathrm{A})$ hold.

REMARK 4. (5.2)(3)(a) provides a probabilistic interpretation of the constants occurring in the Renewal Theorem (3.4).

REMARK 5. (5.2)(3)(b) provides a probabilistic understanding of Theorem (3.6).

REMARK 6. We may give probabilistic versions of the limit theorems for nonnegative $P$-superregular functions:

(5.4) TheOREM. Assume (A) holds. Let $u \geqq 0$ be a P-superregular function. Then $\lim _{k \rightarrow+\infty} u\left(Z_{k}\right)$ exists $\operatorname{Pr}-a . e$. on $\left\{\omega ; \lim _{k \rightarrow \infty} Z_{k}(\omega)=\infty\right\}$. The limit is a finite constant. 


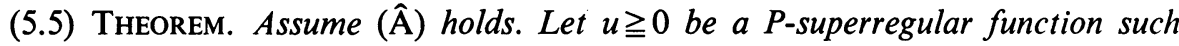
that $\int_{-\infty}(u-P u)(\xi) \mu(d \xi)<\infty$. Then $\lim _{k \rightarrow-\infty} u\left(Z_{k}\right)$ exists Pr-a.e. on

$$
\left\{\omega ; \lim _{k \rightarrow-\infty} Z_{k}(\omega)=-\infty\right\} \text {. }
$$

The limit is a finite constant.

The first theorem is an obvious consequence of results in $\S 2$ and (5.2)(4), the second one of (3.2)(1), (3.5)(3) and (5.2)(4). It is, by the way, easy to see that the integrability condition for $u-P u$ is equivalent to $\sup _{n} \int_{\Omega_{B_{n}}} u\left(Z_{\tau_{B_{n}}}(\omega)\right) d \operatorname{Pr}(\omega)<\infty$ where $B_{n}=(-n, M)$ for some $M \in R$.

6. Proof of the main theorems. First we recall an integral representation for the important $P$-potential $1-\psi$. If $v$ is any $P$-potential, we have by (2.1) and (2.2),

$$
(P v)(\xi)=\int g(\xi, \eta)(v-P v)(\eta) \mu(d \eta), \quad \xi \in R .
$$

This implies for $v=1-\psi$,

$$
\mu(\xi, \xi+1]-\psi(\xi)=\int g(\xi, \eta)\{1-\mu(\eta, \eta+1]\} \mu(d \eta), \quad \xi \in R .
$$

Secondly, we recall from [1] the following notation: For any $P$-superregular function $u \geqq 0$ and any Borel set $A \subseteq R, H_{A} u$ denotes the reduced function of $u$ with respect to $A$.

Proceeding to the proofs of the limit theorems in $\S 3$, we shall start with

(6.3) LemMA. Let $h \geqq 0$ be a finite measurable function on $(-\infty, M+2]$ such that $P h=h$ on $(-\infty, M+1]$. Then $h$ is bounded on $(-\infty, M+1]$.

Proof. Since $h$ is right continuous on $(-\infty, M+1)$ and has left limits on $(-\infty, M+1]$, it is bounded on $[M, M+1]$. Since, by (3.12) in [1] for $\xi \leqq M$, $h(\xi)=H_{(M, M+1\}} h(\xi)$, the lemma follows.

(6.4) Lemma. Assume (Â) does not hold. Let $h$ be as in Lemma (6.3). Then $\lim _{\xi \rightarrow-\infty} h(\xi)=0$.

Proof. Since $h(\xi)=H_{(M, M+1]} h(\xi), \xi \leqq M$, and $h$ is bounded on $(M, M+1]$, it is sufficient to prove that $\lim _{\xi \rightarrow-\infty} H_{(M, M+1]} 1(\xi)=0$. If $(\hat{\mathrm{A}})(\mathrm{i})$ does not hold, this equation is obvious. If $(\hat{\mathrm{A}})(\mathrm{ii})$ does not hold, it follows from

$$
H_{(M, M+1]} 1(\xi) \int_{(\xi+1, M]}\{1-\mu(\eta, \eta+1]\} \mu(d \eta) \leqq 1 \text { for } \xi<M-1,
$$

which one obtains from (6.2) by observing that for $\eta \in(\xi+1, M]$,

$$
H_{(M, M+1]} 1(\xi) \leqq H_{[\eta-1, \eta)} 1(\xi) \leqq G(\xi,[\eta-1, \eta))=g(\xi, \eta) .
$$

(6.5) Lemma. Assume (Â)(i') holds. Let $h$ be as in Lemma (6.3). Let $c$ be a limit point of $h$ as $\xi \rightarrow-\infty$ and let $\delta>0$. If we put $A=\{\xi \leqq M+2 ;|h(\xi)-c|<\delta\}$, then $\int_{-\infty} \chi_{A}(\xi) \mu(d \xi)=\infty$. 
Proof. Let $K \in(\delta / 4, \infty)$ be such that $h(\xi) \leqq K$ for $\xi \in(-\infty, M+1]$. Since $(\hat{A})\left(\mathrm{i}^{\prime}\right)$ holds, there is $L \in R$ such that $\mu(\xi, \xi+1]>1-\delta / 8 K$ for $\xi \leqq L$. Since $c$ is a limit point of $h$ at $-\infty$, there is a sequence $\xi_{n} \downarrow-\infty$ such that $\left|h\left(\xi_{n}\right)-c\right|<\delta / 2$ for all $n$. We may assume that for all $n, \xi_{n} \leqq \operatorname{Min}(M, L)$ and-since $h$ is right continuous on $(-\infty, M+1)$-that $\mu\left\{\xi_{n}\right\}=0$. Define $l_{n} \in(0,1)$ by

$$
l_{n}=\inf \left\{l ; \delta / 8 K<\mu\left(\xi_{n}-l, \xi_{n}\right]\right\} .
$$

Then $\mu\left(\xi_{n}-l_{n}, \xi_{n}\right] \leqq \delta / 8 K$ and $\mu\left[\xi_{n}-l_{n}, \xi_{n}\right] \geqq \delta / 8 K$. Let $A_{1}=\bigcup_{n}\left[\xi_{n}-l_{n}, \xi_{n}\right]$. Then $\int_{-\infty} \chi_{A_{1}}(\xi) \mu(d \xi)=\infty$ because $A_{1}$ contains infinitely many disjoint intervals $\left[\xi_{n}-l_{n}, \xi_{n}\right]$. Moreover $A_{1} \subseteq A$. This is seen as follows: Let $\xi \in\left[\xi_{n}-l_{n}, \xi_{n}\right]$. Then

$$
\begin{aligned}
\left|h(\xi)-h\left(\xi_{n}\right)\right| & \leqq \int_{\left(\xi, \xi_{n}\right]} h(\eta) \mu(d \eta)+\int_{\left(\xi+1, \xi_{n}+1\right]} h(\eta) \mu(d \eta) \\
& \leqq K \mu\left(\xi, \xi_{n}\right]+K \mu\left(\xi+1, \xi_{n}+1\right] .
\end{aligned}
$$

Now

$$
\mu\left(\xi, \xi_{n}\right] \leqq \mu\left(\xi_{n}-l_{n}, \xi_{n}\right] \leqq \delta / 8 K
$$

and

$$
\begin{aligned}
\mu\left(\xi+1, \xi_{n}+1\right] & \leqq \mu\left(\xi, \xi_{n}\right]+\left|\mu(\xi, \xi+1]-\mu\left(\xi_{n}, \xi_{n}+1\right]\right| \\
& \leqq \mu\left(\xi, \xi_{n}\right]+\{1-\mu(\xi, \xi+1]\}+\left\{1-\mu\left(\xi_{n}, \xi_{n}+1\right]\right\} \\
& \leqq \delta / 8 K+\delta / 8 K+\delta / 8 K .
\end{aligned}
$$

Hence $\left|h(\xi)-h\left(\xi_{n}\right)\right| \leqq \delta / 2$. Therefore $|h(\xi)-c|<\delta$, which implies $\xi \in A$. This completes the proof.

(6.6) Lemma. Assume (Â)(i') holds. Let $h$ be as in Lemma (6.3). If $\lim _{\xi \rightarrow-\infty ; \xi \notin A} h(\xi)$ exists for some Borel set $A \subseteq R$ such that $\int_{-\infty} \chi_{A}(\xi) \mu(d \xi)<\infty$, then $\lim _{\xi \rightarrow-\infty} h(\xi)$ exists.

\section{Proof. If}

$$
c=\lim _{\xi \rightarrow-\infty ; \xi \notin A} h(\xi), \quad \int_{-\infty} \chi_{A}(\xi) \mu(d \xi)<\infty,
$$

then $c$ is obviously a limit point of $h$ at $-\infty$. Assume that $h$ has a limit point $c^{\prime} \neq c$ at $-\infty$. By Lemma (6.5) we have $\int_{-\infty} \chi_{A^{\prime}}(\xi) \mu(d \xi)=\infty$ for $A^{\prime}=\{\xi \leqq M+2$; $\left.\left|h(\xi)-c^{\prime}\right|<\left|c-c^{\prime}\right| / 2\right\}$, and hence $\int_{-\infty} \chi_{A_{1}}(\xi) \mu(d \xi)=\infty$ for $A_{1}=\{\xi \leqq M+2 ;|h(\xi)-c|$ $\left.\geqq\left|c-c^{\prime}\right| / 2\right\}$. This contradicts $(*)$, and therefore $\lim _{\xi \rightarrow-\infty} h(\xi)=c$.

We proceed now to the key lemma of this section, which is the only part where we need probabilistic arguments.

(6.7) LemMa. Assume that (Â) holds. Let $h$ be as in Lemma (6.3). Then $\lim _{\xi \rightarrow-\infty ; \xi \notin A} h(\xi)$ exists for some Borel set $A \subseteq R$ such that $\int_{-\infty} \chi_{A}(\xi) \mu(d \xi)<\infty$. 
Proof. In $\S 5$ we introduced an approximate Markov chain on $R$ with transition function $P$ and sojourn measure $\mu$, denoted by $(\Omega, \mathfrak{R}, \operatorname{Pr}) ;\left\{Z_{k},-\infty<k<+\infty\right\}$. For any bounded Borel set $B \subseteq R$, the process $X_{k}^{(B)}=Z_{k+\tau_{B}}, k \geqq 0$, was defined on $\Omega_{B}$.

Now let $B_{n}=(-n, M+1]$ for $n \geqq M$; and on each $\Omega_{B_{n}}$ define the process

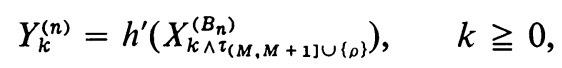

where $\tau_{(M, M+1\} \cup\{\rho\}}$ is the first entry time of $\left\{Z_{k}\right\}$ into $(M, M+1] \cup\{\rho\}$ and $h^{\prime}$ is the extension of $h$ to $(-\infty, M+2] \cup\{\rho\}$ by $h^{\prime}(\rho)=0$. From Theorem (5.2)(2) and Lemma (6.3) we conclude that the functions $Y_{k}^{(n)}$ are integrable, and that their integrals are bounded. Indeed, if $K$ is an upper bound for $h$ on $(-\infty, M+1]$, we have

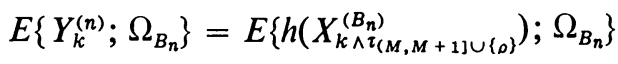

$$
\begin{aligned}
& \leqq K \cdot \operatorname{Pr}\left\{\Omega_{B_{n}}\right\} \leqq K \cdot \operatorname{Pr}\left\{\Omega_{(-\infty, M+1]}\right\}<\infty .
\end{aligned}
$$

From Theorem (5.1)(2), and (3.13) in [1] it follows that the processes $\left\{Y_{k}^{(n)}, k \geqq 0\right\}$, defined on $\Omega_{B_{n}}$, are "martingales". If we now define for $r_{1}, r_{2}$ with $0 \leqq r_{1}<r_{2}<\infty$, $\beta_{n}\left(r_{1}, r_{2}\right)$ on $\Omega_{B_{n}}$ to be the number of upcrossings of $\left\{Y_{k}^{(n)}, k \geqq 1\right\}$ with respect to $\left[r_{1}, r_{2}\right]$, then we have by Doob's celebrated upcrossing inequality

$$
E\left\{\beta_{n}\left(r_{1}, r_{2}\right) ; \Omega_{B_{n}}\right\} \leqq \frac{E\left\{Y_{0}^{(n)} ; \Omega_{B_{n}}\right\}+r_{2} \operatorname{Pr}\left\{\Omega_{B_{n}}\right\}}{r_{2}-r_{1}},
$$

and by $(\times)$ we conclude that $\lim _{n \rightarrow \infty} E\left\{\beta_{n}\left(r_{1}, r_{2}\right) ; \Omega_{B_{n}}\right\}<\infty$. If we define $\beta\left(r_{1}, r_{2}\right)$ on

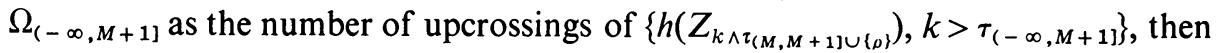
$\beta_{n}\left(r_{1}, r_{2}\right) \chi_{\Omega_{B_{n}}} \uparrow \beta\left(r_{1}, r_{2}\right) \chi_{\Omega_{(-\infty, M+1\}}}$, and we conclude that $E\left\{\beta\left(r_{1}, r_{2}\right) ; \Omega_{(-\infty, M+1\}}\right\}$ $<\infty$. This implies that $\beta\left(r_{1}, r_{2}\right)<\infty$, Pr-a.e. on $\Omega_{(-\infty, M+1 \text { ] }}$, and by a standard argument we obtain

$$
\operatorname{Pr}\left\{\lim _{k \rightarrow-\infty} h\left(Z_{k}\right) \text { exists, } \lim _{k \rightarrow-\infty} Z_{k}=-\infty\right\}=\operatorname{Pr}\left\{\lim _{k \rightarrow-\infty} Z_{k}=-\infty\right\} .
$$

By Theorem $(5.2)\left(4^{\prime}\right)$ there is a $c \in[0, K]$ such that

(+) $\operatorname{Pr}\left\{\lim _{k \rightarrow-\infty} h\left(Z_{k}\right)=c, \lim _{k \rightarrow-\infty} Z_{k}=-\infty\right\}=\operatorname{Pr}\left\{\lim _{k \rightarrow-\infty} Z_{k}=-\infty\right\}$.

The assertion of the lemma follows now by a well-known argument which was also used in [1, p. 234]. For $\varepsilon>0$, let $A_{\varepsilon}=\{\xi \leqq M+2 ;|h(\xi)-c|>\varepsilon\}$. Since (Â) holds, we conclude from $(+)$ and Theorem $(5.2)(4)$ that $\int_{-\infty} \chi_{A_{\varepsilon}}(\xi) \mu(d \xi)<\infty$. Now choose $\xi_{n} \downarrow-\infty, n \geqq 1$, such that $\int \chi_{A_{1 / n} \cap\left(-\infty, \xi_{n}\right)}(\xi) \mu(d \xi)<1 / n^{2}$. Then the assertion of the lemma is true with $A=\bigcup_{n \geqq 1}\left\{A_{1 / n} \cap\left(-\infty, \xi_{n}\right)\right\}$.

Proof of Theorem (3.1). Follows from Lemmas (6.4), (6.6), (6.7).

Proof of Corollary (3.2). Obvious from Theorem (3.1).

Proof of Proposition (3.3). Obvious from Corollary (3.2) and Lemma (4.1) and (4.5) and (4.6)^. 
Remark. Proposition (3.3) is an extension of a formula given by Slater and Wilf [5] in their context.

Proof of Theorem (3.4)(2). We recall that $[P g(\cdot, \eta)](\xi)=g(\xi, \eta)-\chi_{[\eta-1, \eta)}(\xi)$, which implies that $[\operatorname{Pg}(\cdot, \eta)](\xi)=g(\xi, \eta)$ for $\xi<\eta-1$. If $(\hat{\mathrm{A}})$ does not hold, the assertion of the theorem follows from Theorem (3.1). If $(\hat{\mathrm{A}})$ holds, the existence of $\lim _{\xi \rightarrow-\infty} g(\xi, \eta)$ follows from Theorem (3.1); its identification is given by the dual of Theorem (6.5) in [1], but can also be achieved from

$$
\mu[\eta-1, \eta)-\hat{\psi}(\eta)=\int\{1-\mu[\xi-1, \xi)\} \hat{g}(\eta, \xi) \mu(d \xi)
$$

by (2.3) and Lemma (4.1) and (4.6)^, since $g(\xi, \eta)=0$ for $\xi \geqq \eta$ and

$$
\int\{g(\xi, \eta)-[P g(\cdot, \eta)](\xi)\} \mu(d \xi)=\mu[\eta-1, \eta) .
$$

\section{Proof of Theorem (3.5).}

(1) This assertion follows from (6.1) and (3.4)(2) by the dominated convergence theorem.

(2) We have for $M \in R, P v(\xi) \geqq \int_{(-\infty, M)} g(\xi, \eta) f(\eta) \mu(d \eta)$, which implies that

$$
\liminf _{\xi \rightarrow-\infty} P v(\xi) \geqq \frac{2}{1+\hat{\alpha}} \int_{(-\infty, M)} \hat{\psi}(\eta) f(\eta) \mu(d \eta)
$$

The assertion follows because $\lim _{\eta \rightarrow-\infty} \hat{\psi}(\eta)=1$.

(3) It is sufficient to prove that

$$
\lim _{\xi \rightarrow-\infty} P v(\xi)=\frac{2}{1+\hat{\alpha}} \int \hat{\psi}(\eta) f(\eta) \mu(d \eta)<\infty,
$$

since $\int_{-\infty} f(\eta) \mu(d \eta)<\infty$ implies that $\lim _{\xi \rightarrow-\infty ; \xi \notin A} f(\xi)=0$ for some Borel set $A \subseteq R$ such that $\int_{-\infty} \chi_{A}(\eta) \mu(d \eta)<\infty$. Now let $M \in R$. From (3.4)(2) and the dominated convergence theorem it is clear that

$$
\lim _{\xi \rightarrow-\infty} \int_{(-\infty, M]} g(\xi, \eta) f(\eta) \mu(d \eta)=\frac{2}{1+\hat{\alpha}} \int_{(-\infty, M]} \hat{\psi}(\eta) f(\eta) \mu(d \eta)<\infty .
$$

In view of $(6.1)$ it is therefore sufficient to prove that

$$
\text { (-) } \quad \lim _{\xi \rightarrow-\infty} \int_{(M, \infty)} g(\xi, \eta) f(\eta) \mu(d \eta)=\frac{2}{1+\hat{\alpha}} \int_{(M, \infty)} \hat{\psi}(\eta) f(\eta) \mu(d \eta)<\infty .
$$

If $\xi \leqq M-1$, we have for $\eta>M, g(\xi, \eta)=\hat{g}(\eta, \xi) \leqq 4 \hat{H}_{(M-1, M]} 1(\eta) \leqq 4 \hat{g}(\eta, M-1)$ $=4 g(M-1, \eta)$. (The first inequality follows from a slight modification of (3.12) in [1].) But since

$$
\int_{(M, \infty)} g(M-1, \eta) f(\eta) \mu(d \eta) \leqq \int_{(M-1, \infty)} g(M-1, \eta) f(\eta) \mu(d \eta)=P v(M-1)<\infty,
$$

(-) follows from Theorem (3.4)(2) and the dominated convergence theorem. 
Proof of Theorem (3.6) If either (A) or (Â) do not hold, we have $\psi(-\infty)$ $=\hat{\psi}(+\infty)=0$. We assume now that both (A) and ( $\hat{\mathrm{A}})$ hold. By the dual of Proposition (3.3) we have

$$
\int \hat{\psi}(\xi)\{1-\mu(\xi, \xi+1]\} \mu(d \xi) \doteq \frac{1}{2}(1+\hat{\alpha})-(\hat{\psi}(\infty) / 2)(1+\alpha) .
$$

On the other hand, we get from (6.2) and Theorem (3.4)(2)

$$
\frac{2}{1+\hat{\alpha}} \int \hat{\psi}(\eta)\{1-\mu(\eta, \eta+1]\} \mu(d \eta)=1-\psi(-\infty) .
$$

Therefore, $\hat{\psi}(\infty)(1+\alpha)=\psi(-\infty)(1+\hat{\alpha})$, and the proof is complete.

\section{BIBLIOGRAPHY}

1. G. A. Brosamler, Potential theoretic analysis of a certain integral equation, Trans. Amer. Math. Soc. 129 (1967), 218-248. MR 36 \#2226.

2. G. A. Hunt, Markov chains and Martin boundaries, Illinois J. Math. 4 (1960), 313-340. MR 23 \#A691.

3. J. G. Kemeny, J. L. Snell and A. W. Knapp, Denumerable Markov chains, Van Nostrand, Princeton, N. J., 1966. MR 34 \#6858.

4. G. Placzek, On the theory of slowing down of neutrons in heavy substances, Phys. Rev. (2) 69 (1946), 423-438. MR 8, 30.

5. M. L. Slater and H. S. Wilf, A class of linear differential-difference equations, Pacific J. Math. 10 (1960), 1419-1427. MR 23 \#A3329.

6. M. L. Slater, On the equation $\phi(x)=\int_{x}^{x+1} K(\xi) f[\phi(\xi)] d \xi$, Pacific J. Math. 20 (1967), 155166. MR 34 \#6471.

Department of Mathematics, University of British Columbia, Vancouver, British Columbia, Canada 